\title{
Analytical, physiologic, and clinical validation of a radioimmunoassay for measurement of procollagen type III amino terminal propeptide in serum and bronchoalveolar lavage fluid obtained from dogs
}

\author{
Simone Schuller, DVM; Sophie Valentin, DVM; Benoit Remy, DVM; Pascale Jespers; \\ Suzanne Foulon; Nicole Van Israël, DVM; Cécile Clercx, DVM, PhD; Kathleen McEntee, DVM, PhD
}

\begin{abstract}
Objective-To validate a radioimmunoassay for measurement of procollagen type III amino terminal propeptide (PIIINP) concentrations in canine serum and bronchoalveolar lavage fluid (BALF) and investigate the effects of physiologic and pathologic condi-
\end{abstract} tions on PIIINP concentrations.

Sample Population-Sera from healthy adult $(n=70)$ and growing dogs (20) and dogs with chronic renal failure (CRF; 10), cardiomyopathy (CMP; 12), or degenerative valve disease (DVD; 26); and sera and BALF from dogs with chronic bronchopneumopathy (CBP; 15) and healthy control dogs (10 growing and 9 adult dogs).

Procedure - A radioimmunoassay was validated, and a reference range for serum PIIINP (S-PIIINP) concentration was established. Effects of growth, age, sex, weight, CRF, and heart failure on S-PIIINP concentration were analyzed. In CBP-affected dogs, S-PIIINP and BALF-PIIINP concentrations were evaluated.

Results-The radioimmunoassay had good sensitivity, linearity, precision, and reproducibility and reasonable accuracy for measurement of S-PIIINP and BALF-PIIINP concentrations. The S-PIIINP concentration reference range in adult dogs was 8.86 to 11.48 $\mu \mathrm{g} / \mathrm{L}$. Serum PIIINP concentration correlated with weight and age. Growing dogs had significantly higher S-PIIINP concentrations than adults, but concentrations in CRF-, CMP-, DVD-, or CBP-affected dogs were not significantly different from control values. Mean BALF-PIIINP concentration was significantly higher in CBP-affected dogs than in healthy adults.

Conclusions and Clinical Relevance-In dogs, renal or cardiac disease or CBP did not significantly affect S-PIIINP concentration; dogs with CBP had high BALFPIIINP concentrations. Data suggest that the use of PIIINP as a marker of pathologic fibrosis might be limited in growing dogs. (Am J Vet Res 2006;67:749-755)

Received October 7, 2005

Accepted November 28, 2005.

From the Departments of Small Animal Clinical Sciences, (Schuller, Valentin, Van Israël, Clercx) and Physiology of Reproduction (Remy), University of Liège, 4000 Liège, Belgium; and the Laboratory of Physiology, Free University of Brussels, 1070 Brussels, Belgium (Jespers, Foulon, McEntee). Dr Schuller's present address is Department for Small Animal Clinical Studies, University College Dublin, Belfield, Dublin 4, Ireland.

Presented in part at the 14th Congress of the European College of Veterinary Internal Medicine, Barcelona, Spain, September 2004, and at the 15th Congress of the European College of Veterinary Internal Medicine, Glasgow, September 2005.

Address correspondence to Dr. Clercx.

\begin{tabular}{lll}
\hline \multicolumn{2}{c}{ ABbreviations } \\
PIIINP & $\begin{array}{l}\text { Procollagen type III amino terminal } \\
\text { propeptide }\end{array}$ \\
BALF & $\begin{array}{l}\text { Bronchoalveolar lavage fluid } \\
\text { CMP }\end{array}$ & Cardiomyopathy \\
DVD & Degenerative valve disease \\
CRF & Chronic renal failure \\
CBP & Chronic bronchopneumopathy \\
ED & Effective dose \\
IGF-I & Insulinlike growth factor-I
\end{tabular}

M any chronic diseases in humans and animals are associated with modifications of the extracellular matrix metabolism that lead to an accumulation of collagen and the development of organ fibrosis. ${ }^{1}$ For a long time, histologic examination of a tissue biopsy specimen was the only available method to evaluate organ fibrosis. The need for less invasive, more dynamic, and more quantitative tests has led to the identification of several markers of extracellular matrix turnover, one of which is PIIINP. ${ }^{2}$ During synthesis of type III collagen, PIIINP is cleaved off the procollagen type III molecule in a stoichiometric manner. ${ }^{3}$ It has been studied extensively in many human biological fluids including serum ${ }^{4}$ and $\mathrm{BALF}^{5}$ and has been established as a sensitive but nonspecific marker for assessment of tissue collagen type III turnover. ${ }^{6}$

Procollagen type III amino terminal propeptide has been widely investigated in several physiologic and pathologic conditions in humans. ${ }^{7}$ In healthy children, serum PIIINP concentration is significantly higher than in adults and the highest values are detected during the neonatal period. ${ }^{8}$ Serum PIIINP concentration closely parallels the growth-velocity curve of children, reflecting the increased collagen turnover during growth. There are no significant sex-related differences in serum PIIINP concentration. ${ }^{9}$ In humans, increases in serum PIIINP concentration have been identified in several hepatic disorders including chronic hepatitis, ${ }^{10}$ alcoholic liver disease, ${ }^{11}$ and other fibrosing disorders such as myelofibrosis ${ }^{12}$ and systemic sclerosis. ${ }^{13}$ Increased serum PIIINP concentration has been determined in various malignancies and correlates with the clinical extent and behavior of the tumor. ${ }^{14}$

Serum PIIINP concentration has been proven to reliably reflect increased collagen type III turnover associated with cardiac tissue repair and fibrosis and to 
be of prognostic value in many conditions such as myocardial infarction ${ }^{15}$ as well as various types of cardiomyopathy. ${ }^{16}$ In patients with severe congestive heart failure, serum PIIINP concentration is closely correlated with clinical stage and outcome. ${ }^{17}$ Serum and urinary PIIINP concentrations have been studied in humans with various renal diseases ${ }^{18,19}$ as well as in patients after renal transplantation ${ }^{20}$ and have been shown to correlate with the extent of organ fibrosis. In interstitial pulmonary conditions including pulmonary fibrosis, ${ }^{5}$ acute respiratory distress syndrome $e^{21}$ and sarcoidosis, ${ }^{22}$ PIIINP concentrations in both serum and BALF are significantly increased, compared with values in samples from healthy individuals.

The aims of the study reported here were to validate a radioimmunoassay for measurement of PIIINP in canine serum and BALF and investigate the effects of different physiologic and pathologic conditions on PIIINP concentrations. The intentions were to establish a new tool for noninvasive investigation of extracellular matrix turnover in dogs for both clinical and research application and evaluate the influences of growth, age, sex, weight, CMP, DVD, CRF, and CBP on PIIINP concentration in canine serum and BALF.

\section{Materials and Methods}

For this study, 2 populations of dogs (overall, 172 dogs) were used. Serum samples from 138 dogs, including healthy adults $(\mathrm{n}=70)$, healthy growing dogs $(20)$, dogs with chronic heart disease (CMP, 12; DVD, 26), and CRF (10) were collected prospectively. The reference range for serum PIIINP concentration and the influence of growth, age, weight, and sex were examined in this group of 138 dogs. For the evaluation of PIIINP concentrations in dogs with CBP, leftover serum and BALF samples from 13 dogs with eosinophilic BP and 2 dogs with chronic bronchitis that had participated in another clinical trial and their respective control dogs (9 healthy adults and 10 healthy growing dogs) were used. Dogs included in the study were owned by students, staff, or clients of the small animal hospital of the Faculty of Veterinary Medicine of Liège, Belgium. Fourteen healthy adult and 11 healthy growing Beagles, belonging to the Beagle colony of the hospital, were also included in the study. Enrollment was based on owner consent. Dogs were considered healthy if they did not have a history of clinical disease as reported by the owners and no abnormalities were detected during physical examination. However, further diagnostic tests to establish their health status were not performed. In dogs with CMP and DVD, diagnosis was made on the basis of history; clinical signs; and compatible results of physical examination, thoracic radiography, ECG, and echocardiography. The diagnosis of CRF was made on the basis of history; clinical signs; and compatible results of physical examination, blood analyses, urinalysis, and abdominal ultrasonography. In dogs with CBP, diagnosis was made on the basis of history, clinical signs, findings of physical examination and thoracic radiography, and results of bronchoscopy and BALF analysis (including cytologic and microbiologic evaluations).

Serum and BALF sample collections-Venous blood samples were collected and placed into plastic tubes containing a separation gel. ${ }^{a}$ The tubes were centrifuged $\mathrm{d}^{\mathrm{b}}$; the serum was transferred into separate tubes $s^{c}$ and frozen at $-20^{\circ} \mathrm{C}$ until analysis. The leftover serum samples from dogs with CBP and the respective control dogs were obtained in the same manner but frozen in the original tubes containing the separation gel. Each BALF sample had been obtained during bronchoscopic examination by use of a standardized method described elsewhere..$^{23}$ The BALF samples were centrifuged immediately after retrieval and then stored at $-20^{\circ} \mathrm{C}$ until analysis.

Procedure for the PIIINP radioimmunoassay-Serum and BALF concentrations of PIIINP were measured by use of a commercially available PIIINP radioimmunoassay ${ }^{d}$ that was based on highly purified human PIIINP. The assays were performed according to the procedure indicated in the instruction booklet with 3 slight modifications. The first modification was a reduction of the volumes of samples and reagents by $50 \%$ to increase the number of samples processed per kit. The second modification was the introduction of supplementary dilution of the samples before centrifugation with $1 \mathrm{~mL}$ of PBS solution ${ }^{e}$ to facilitate aspiration of the supernatant and reduce the concentration of iodine $125\left({ }^{125} \mathrm{I}\right)$-labeled PIIINP in the supernatant retained in the tube after aspiration. The third modification consisted of a slight prolongation of centrifugation time from 15 to 25 minutes and increased centrifugation speed from $2,000 \times g$ to $2,250 \times g$ to achieve better separation of supernatant and precipitate and increase the stability of the precipitate. For analysis of BALF samples, $1 \mathrm{~mL}$ of BALF or standard solution was incubated with $200 \mu \mathrm{L}$ of ${ }^{125} \mathrm{I}$-tracer solution and $100 \mu \mathrm{L}$ of antiserum to detect small amounts of PIIINP in the samples without further concentration.

Validation of the PIIINP radioimmunoassay-The assay was validated by determining its sensitivity, working range, linearity, accuracy, precision, and reproducibility. Serial dilutions of a serum sample by use of saline $(0.9 \%$ $\mathrm{NaCl}$ ) solution ${ }^{\mathrm{f}}$ or the zero calibrator of the kit were performed. The sample was assayed undiluted and at dilutions of 1 in 2, 1 in 3,1 in 4,1 in 5,1 in 6,1 in 8,1 in 10,1 in 12, and 1 in 16 . For evaluation of parallelism of the standard curves, concentrations of the standard solutions were plotted with a semilogarithmic scale on the $\mathrm{x}$-axis, whereas values on the $y$-axis were expressed as a percentage of the maximum binding value by use of the following equation:

$$
\mathrm{B} / \mathrm{B}_{0}=\left(\mathrm{B}-\mathrm{B}_{0}\right) \bullet 100 /\left(\mathrm{B}_{\max }-\mathrm{B}_{0}\right)
$$

where $B$ is the sample (or calibrator) count, $B_{0}$ is the nonspecific-binding count, and $\mathrm{B}_{\max }$ is the total count. The EDs, which are defined as the calibrator concentration corresponding to $20 \%\left(\mathrm{ED}_{20}\right), 50 \%\left(\mathrm{ED}_{50}\right)$, and $80 \%\left(\mathrm{ED}_{80}\right)$ inhibition of the standard curve, were determined. For evaluation of dilution parallelism, the PIIINP concentrations were plotted as a semilogarithmic curve and the observed PIIINP concentrations were compared with the expected values. Spiking recovery was determined by adding concentrations of human PIIINP $(1,2.5,5,7.5$, and $10 \mu \mathrm{g} / \mathrm{L})$ to a canine serum sample with a known PIIINP concentration (6.6 $\mu \mathrm{g} / \mathrm{L})$. Observed PIIINP concentrations were compared with the expected values.

Inter- and intra-assay variabilities were determined by use of serum samples with 3 different PIIINP concentrations (low, $4.03 \mu \mathrm{g} / \mathrm{L}$; midrange, $11.48 \mu \mathrm{g} / \mathrm{L}$; and high, $15.69 \mu \mathrm{g} / \mathrm{L}$ ). For calculation of the intra-assay variability, each of the 3 serum samples was divided in 9 replicates. The replicates were assayed simultaneously within the same run of the assay. For calculation of the interassay variability, each of the 3 samples was divided in 6 replicates and assayed in consecutive runs of the assay during a 9-month period.

Stability of PIIINP during freezing and thawing-To determine the stability of PIIINP in canine serum, aliquots of samples containing 3 concentrations of PIIINP were run through 6 freeze-thaw cycles over a period of 9 months. After every cycle, serum PIIINP concentrations were measured and compared with each other. 
Statistical analysis-Statistical evaluation was performed by use of personal computer-based statistical software. Normal distribution of the data was determined by producing a normal plot. The reference range for serum PIIINP concentration in adult dogs was established on the basis of the $95 \%$ confidence interval for the mean serum PIIINP concentration in healthy adult dogs. An ANOVA, followed by an unpaired $t$ test, was used to evaluate the following independent variables with regard to PIIINP concentration: weight, age, sex, DVD, CMP, CRF, and CBP. Multivariate linear regression analysis was used to assess the relationship between serum PIIINP concentration and weight after adjustment for age. Serum PIIINP concentrations of dogs with heart disease and CRF were compared with weight- and age-matched control dogs. Mean PIIINP serum concentrations were compared between healthy growing Beagles and growing dogs of other breeds and between adult healthy Beagles and weight- and age-matched dogs of other breeds, given that Beagles were overrepresented in the group of healthy dogs. Statistical sig- nificance was defined as a value of $P<0.05$. Data are expressed as mean \pm SD unless otherwise stated.

\section{Results}

Study population-One hundred seventy-two dogs were included in the study. In each study group, sex; breeds; and mean values of age, weight, and PIIINP concentrations in serum and BALF were assessed (Tables 1 and 2). In the group of dogs with heart disease, 26 dogs had DVD of the mitral $(n=23)$ or mitral and tricuspid valve (3). In the group of dogs with CMP primary dilatated CMP was diagnosed in 11 dogs and arrhythmogenic right ventricular CMP was diagnosed in 1 dog. Dogs with CRF were in an advanced stage (serum creatinine concentration $>3 \mathrm{mg} / \mathrm{dL}$; reference range, 0.68 to $1.45 \mathrm{mg} / \mathrm{dL}$ ); proteinuria (urine proteinto-urine creatinine ratio $>1$ ) was identified in 2 of the

Table 1-Signalment and mean serum PIIINP concentrations in healthy dogs and dogs with cardiac or renal disease.

\begin{tabular}{|c|c|c|c|c|c|}
\hline \multirow[b]{2}{*}{ Variable } & \multicolumn{2}{|l|}{ Healthy } & \multicolumn{3}{|c|}{ Dogs with disease } \\
\hline & Adult dogs & Growing dogs & DVD & CMP & CRF \\
\hline $\begin{array}{l}\text { No. of dogs } \\
\text { Sex (No of dogs) }\end{array}$ & 70 & 20 & 26 & 12 & 10 \\
\hline $\begin{array}{l}\text { Male/neutered male } \\
\text { Female/spayed female }\end{array}$ & $\begin{array}{l}21 / 9 \\
15 / 13\end{array}$ & & $\begin{array}{c}13 \\
1 / 12\end{array}$ & $\begin{array}{l}10 \\
1 / 1\end{array}$ & $\begin{array}{c}7 \\
1 / 2\end{array}$ \\
\hline $\begin{array}{l}\text { Age (mean } \pm \text { SD } \\
\text { [range]; y) }\end{array}$ & $5.9 \pm 3.1(1-15.5)$ & $0.3 \pm 0.2(0.1-0.6)$ & $10.5 \pm 2.8(4-15)$ & $8.5 \pm 2.8(4-12)$ & $6.5 \pm 2.2(3-11)$ \\
\hline $\begin{array}{l}\text { Weight (mean } \pm \text { SD } \\
\text { [range]; kg) }\end{array}$ & $21.1 \pm 13.5(2.5-60)$ & $8.4 \pm 3.4(4.5-15)$ & $10.8 \pm 8.6(2.4-46)$ & $37 \pm 15.7(14.7-70)$ & $31.6 \pm 5.7(25-40)$ \\
\hline Breeds (No. of dogs) & $\begin{array}{l}\text { Beagle (15) } \\
\text { Mixed (14) } \\
\text { Yorkshire Terrier (5) } \\
\text { Labrador Retriever (4) } \\
\text { Dachshund (3) } \\
\text { Griffon Kortals (3) } \\
\text { German Shepherd Dog (3) } \\
\text { Other purebreds (23) }\end{array}$ & $\begin{array}{l}\text { Beagle (11) } \\
\text { Labrador Retriever (2) } \\
\text { Mixed (2) } \\
\text { Other purebreds (5) }\end{array}$ & $\begin{array}{l}\text { Dachshund (5) } \\
\text { Maltese (5) } \\
\text { Poodle (4) } \\
\text { Mixed (3) } \\
\text { Other purebreds (9) }\end{array}$ & $\begin{array}{l}\text { Labrador Retriever (3) } \\
\text { Mixed (2) } \\
\text { Great Dane (1) } \\
\text { Cocker Spaniel (1) } \\
\text { Boxer (1) } \\
\text { German Shepherd } \\
\text { Dog (1) } \\
\text { Newfoundland (1) } \\
\text { Other purebreds (2) }\end{array}$ & $\begin{array}{l}\text { Belgian Shepherd } \\
\text { Dog (2) } \\
\text { Labrador Retriever (2) } \\
\text { Bernese Mountain } \\
\text { Dog (2) } \\
\text { Mixed (2) } \\
\text { Other purebreds (2) }\end{array}$ \\
\hline $\begin{array}{l}\text { Serum PIIINP } \\
\text { (mean } \pm \text { SD [range]; } \\
\mu \mathrm{g} / \mathrm{L})\end{array}$ & $10.2 \pm 5.5(2.5-26.1)$ & $106.8 \pm 67.9(17.7-252)$ & $10.5 \pm 2.8(2.5-15.5)$ & $9.6 \pm 2.8(6.5-14.8)$ & $14.9 \pm 1.5(8-23.1)$ \\
\hline
\end{tabular}

Table 2-Signalment and mean PIIINP concentrations in serum and BALF in healthy adult and growing dogs and dogs with CBP.

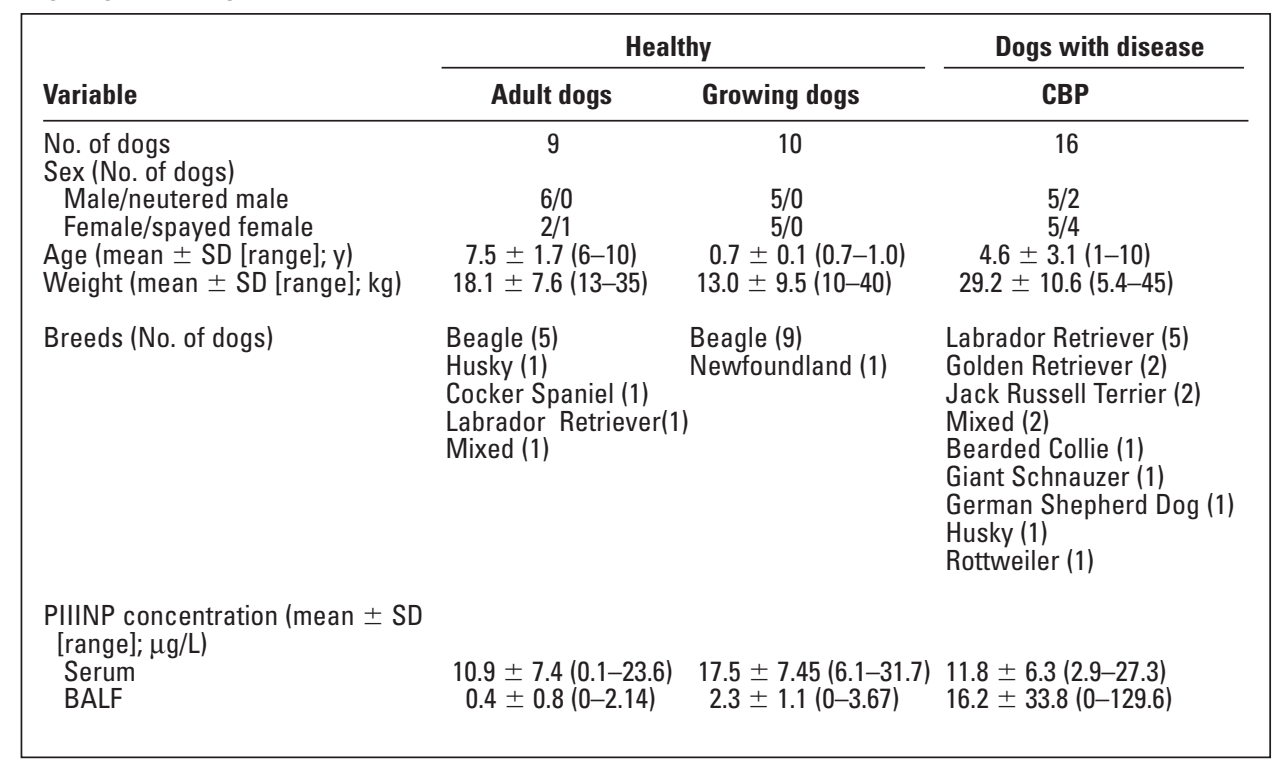


10 dogs in this group. In the group of dogs with CBP, 13 dogs had evidence of eosinophilic BP and chronic bronchitis was diagnosed in 2 dogs.

Validation of the PIIINP radioimmunoassayStandard inhibition curves of human standard solutions and serial dilutions of a canine serum sample were similar and had good linearity and parallelism (Figure 1). The $\mathrm{ED}_{20}, \mathrm{ED}_{50}$, and $\mathrm{ED}_{80}$ were $17.49 \mu \mathrm{g} / \mathrm{L}$, $4.4 \mu \mathrm{g} / \mathrm{L}$, and $0.99 \mu \mathrm{g} / \mathrm{L}$, respectively. Reproducible linear results for the logarithmically transformed dilution curves were obtained. Strong correlation was determined between measured and calculated values for both diluents, saline solution and zero calibrator (Figure 2). The observed-to-expected ratio for spiking recovery ranged from $87 \%$ to $121 \%$ (mean \pm SD recovery, $114.1 \pm 7.14 \%$ ). In 3 serum samples with low, midrange, or high PIIINP concentration, coefficients of variation for intra-assay variability were 5.34\%, 6.42\%, and $5.82 \%$, respectively, and coefficients of variation for interassay variability were $24.74 \%, 5.68 \%$, and $15.71 \%$, respectively. Serum PIIINP concentrations of 3 samples did not vary significantly $(P=0.5)$ when run through 6 freeze-thaw cycles.

Serum PIIINP concentrations in healthy dogsThe reference range for serum PIIINP concentration in healthy adult dogs was 8.27 to $11.7 \mu \mathrm{g} / \mathrm{L}$ (mean, 9.894 $\mu \mathrm{g} / \mathrm{L}$ [SD, $5.06 \mu \mathrm{g} / \mathrm{L} ; \mathrm{SEM}, 0.609 \mu \mathrm{g} / \mathrm{L}])$. Mean serum PIIINP concentration was significantly $(P<0.01)$ higher in growing dogs (0 to 8 months old) than in adult dogs ( $>12$ months old). Mean serum PIIINP concentrations were highest in dogs $<2$ months old $(208.79 \pm 32.3 \mu \mathrm{g} / \mathrm{L})$ and decreased by $50 \%$ at the age of 4 months $(100.07 \pm 24.3 \mu \mathrm{g} / \mathrm{L})$. A further $50 \%$ decrease was detected at the age of 8 months $(54.57 \pm$ $21.3 \mu \mathrm{g} / \mathrm{L})$. There was a mild negative correlation between age and serum PIIINP concentration $\left(R^{2}=\right.$

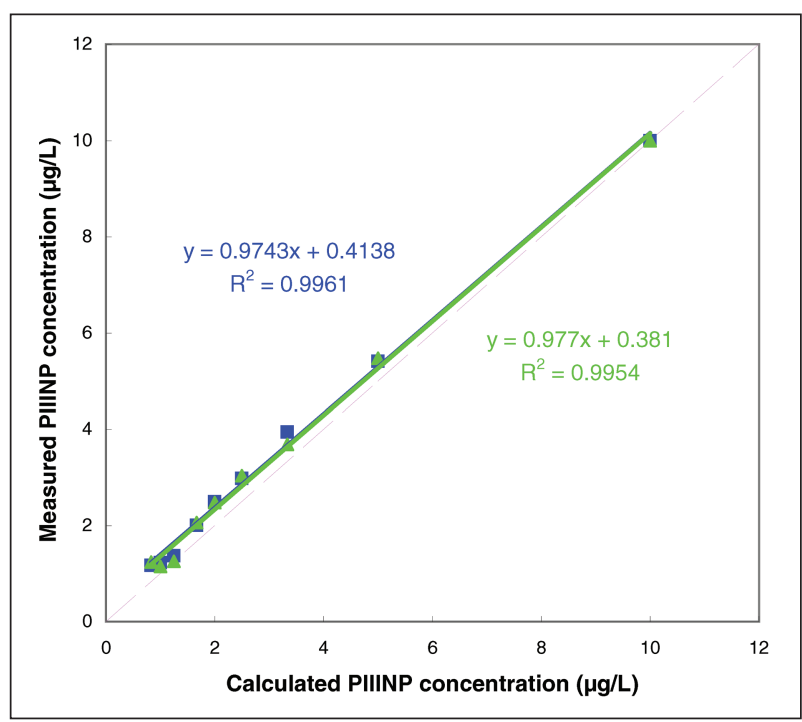

Figure 1-Dilution curve of a canine serum sample diluted with saline $(0.9 \% \mathrm{NaCl})$ solution (triangles) or the zero calibrator (squares) provided in the kit of a commercially available radioimmunoassay for PIIINP. The results are plotted as semilogarithmic curve. Notice the strong correlation between the measured and calculated PIIINP values for samples diluted with the zero calibrator or saline solution.
$0.121 ; P<0.05)$ and a positive correlation between weight and serum PIIINP concentration $\left(R^{2}=0.345 ; P\right.$ $<0.001)$. No correlation was evident between sex and serum PIIINP concentration. Mean serum PIIINP concentration was not significantly $(P=0.72)$ different between growing Beagles $(76.46 \pm 15.71 \mu \mathrm{g} / \mathrm{L})$ and growing dogs of other breeds $(70.09 \pm 9.53 \mu \mathrm{g} / \mathrm{L})$ nor between adult healthy Beagles (6.20 $\pm 0.99 \mu \mathrm{g} / \mathrm{L})$ and weight- and age-matched healthy dogs of other breeds $(6.98 \pm 0.63 \mu \mathrm{g} / \mathrm{L} ; P=0.51)$.

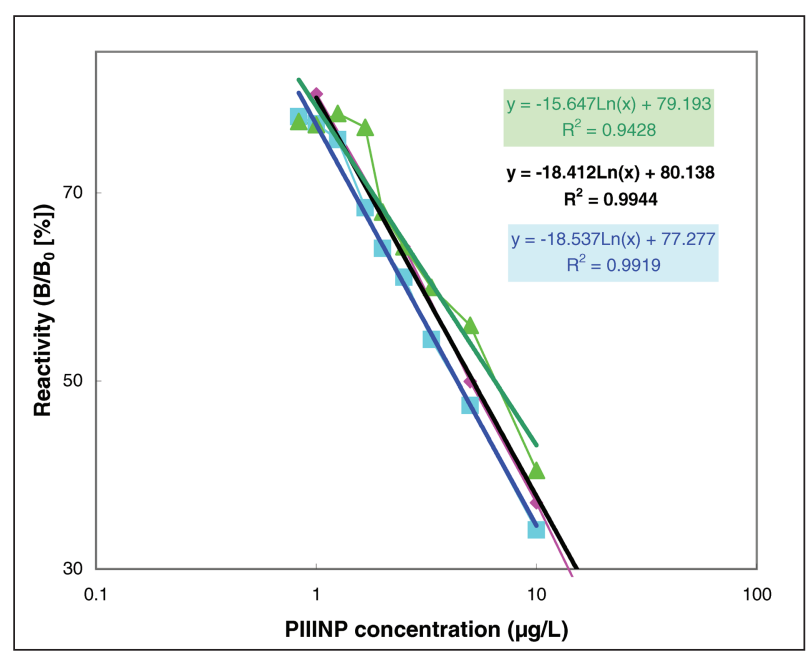

Figure 2-Parallelism of standard inhibition curves of a canine serum sample diluted with saline solution (triangles) or the zero calibrator (squares) provided in the kit of a commercially available radioimmunoassay for PIIINP. Good linearity and strong correlation between calibration curve (diamonds) and standard inhibition curves for samples diluted with the zero calibrator or saline solution. $\mathrm{B}=$ Sample (or calibrator) count. $\mathrm{B}_{0}=$ Nonspecific-binding count.

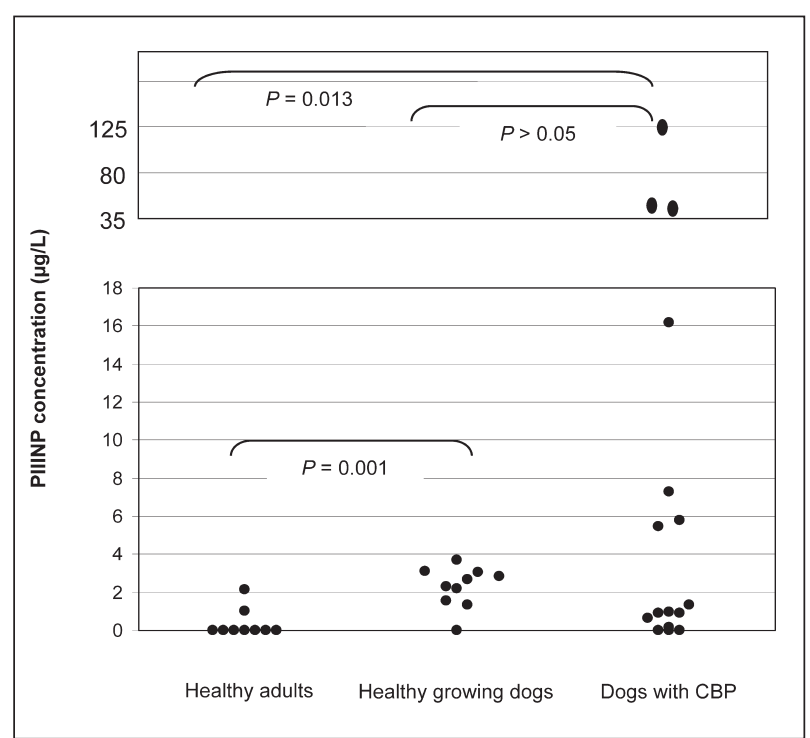

Figure 3-Comparison of PIIINP concentrations in BALF samples collected from 9 healthy adult dogs, 10 healthy growing dogs, and 15 dogs with CBP. Mean BALF PIIINP concentration was significantly higher in growing dogs than in healthy adult dogs. Mean BALF PIIINP concentration was significantly higher in dogs with CBP than in healthy adult dogs. There was no significant difference in mean BALF PIIINP concentration between dogs with CBP and healthy growing dogs. A value of $P<0.05$ was considered significant. 
Serum PIIINP concentrations in dogs with heart disease and CRF-There were no significant differences in serum PIIINP concentration in dogs with DVD, CMP, or CRF, compared with weight- and agematched control dogs.

Serum and BALF PIIINP concentrations in dogs with CBP-Serum PIIINP concentration in dogs with CBP was not significantly different from that of adult control dogs, but the difference in serum PIIINP concentration between dogs with CBP and healthy growing dogs was significant. Mean PIIINP concentration in BALF was significantly higher in healthy growing dogs than in healthy adults (Figure 3). Mean PIIINP concentration in BALF of dogs with CBP was significantly higher than the value in healthy adult dogs, but not significantly different from mean BALF PIIINP concentration in growing dogs. Procollagen type III amino terminal propeptide was undetectable in BALF samples from 7 of 9 healthy adult dogs and from only 3 of 16 dogs with CBP. Nevertheless, there was considerable overlap between the 2 groups.

\section{Discussion}

Procollagen type III amino terminal propeptide is considered a sensitive but nonspecific marker for assessment of tissue collagen III metabolism in humans. ${ }^{6}$ The aims of the present study were to validate a commercially available radioimmunoassay that was based on highly purified human PIIINP for use in dogs and to investigate the effects of different physiologic and pathologic conditions on PIIINP concentration in canine serum and BALF. The radioimmunoassay used is very sensitive and precise for measurement of PIIINP in many human biological fluids including serum ${ }^{9}$ and BALF. $^{22}$ The results of the present study indicated that the assay has good sensitivity, linearity, and reproducibility and acceptable accuracy for measurement of PIIINP in canine serum. Good cross-reactivity between human and canine PIIINP could be expected, given that collagen synthesis and metabolism are known to be well preserved throughout many mammalian species. ${ }^{24}$

Spiking recovery was $87 \%$ to $121 \%$ (mean recovery, $114.1 \pm 7.14 \%$ ), which we consider a satisfactory result. Intra- and interassay variations are indicators of assay precision, and coefficients of variation $<10 \%$ to $15 \%$ are usually set as an acceptable standard. In the present study, the intra-assay coefficient of variation for the 9 replicates of the 3 samples containing low, midrange, or high PIIINP concentration were $<7 \%$, indicating excellent precision. The interassay coefficients of variation for the 6 replicates of the same 3 samples were $5.68 \%$ for the midrange PIIINP concentration and $15.71 \%$ for the high PIIINP concentration, but $24.7 \%$ for the low PIIINP concentration, which might indicate limited precision at the low and the high working ranges of the assay. However, in our opinion, the overall precision of the assay can still be considered good. Furthermore, serum PIIINP concentrations were not significantly altered after 6 freezethaw cycles; these results correspond with findings for human PIIINP, which is considered to be very stable in serum. ${ }^{25}$
Among the dogs used in our study, there were no sex-related differences in serum PIIINP concentrations; however, the influence of neutering on serum PIIINP concentration remains to be analyzed. The serum PIIINP concentration in Beagles was not significantly different from that in other breeds. Mean serum PIIINP concentration was approximately 10 to 20 times as high in growing dogs as it was in adult dogs; the values followed the dogs' growth curve, and adult serum PIIINP concentrations were attained at approximately 10 to 12 months. These results are consistent with the values reported in children ${ }^{8}$ and earlier results in German Shepherd Dog puppies. ${ }^{26}$ The results of the present study indicated that not only the mean serum PIIINP concentration but also the mean BALF PIIINP concentration was significantly higher in healthy growing dogs than in healthy adult dogs, thus underlining the fact that the amount of PIIINP reflects the extent of collagen turnover during growth. The naturally higher concentrations of PIIINP detected in serum and BALF samples from dogs during growth might limit the use of PIIINP as a marker of pathologic fibrosis in growing individuals because high PIIINP concentrations may mask any alteration related to disease.

In the present study, serum PIIINP concentration was negatively correlated with age. This finding is intriguing because the opposite seems to be true in humans. In humans, a slight increase of serum PIIINP concentration after the age of 50 has been reported. ${ }^{27}$ We also identified a positive correlation of serum PIIINP concentration with body weight. This correlation was highly significant and confirmed via multiple linear regression analysis after adjustment for age. In humans, changes in serum PIIINP concentration reflect an increased collagen turnover in response to increases in concentrations of growth hormone and IGF-I; serum PIIINP concentration has been proposed as a marker for monitoring the response to growth hormone therapy in growth hormone-deficient children, ${ }^{28}$ as well as for detection of growth hormone abuse in athletes. ${ }^{29}$ In dogs, serum IGF-I concentration is significantly correlated with weight ${ }^{30}$; therefore, high serum PIIINP concentration might reflect higher IGF-I concentrations in large-breed dogs. To the authors' knowledge, no relationship between body size and serum PIIINP concentration in humans has been reported, but there is a positive correlation between serum PIIINP concentration and weight in healthy obese people. Interestingly, serum PIIINP concentration decreases during weight loss in these individuals. ${ }^{31}$ The influence of obesity on serum PIIINP concentration in dogs has not been addressed so far but merits further investigation.

Compared with weight- and age-matched control dogs, serum PIIINP concentration in dogs with DVD, CMP, or CRF did not differ significantly; similarly, values in dogs with CBP and healthy control dogs did not differ. These negative results have not yet been explained, but could be attributable to several factors given that serum PIIINP concentration depends not only on collagen type III production by the tissue of origin but also on the rate of release of the propeptide into the bloodstream and its clearance by the liver. ${ }^{32}$ 
A limitation of the present study is the fact that organ fibrosis was not confirmed by histologic examination of tissue biopsy specimens, which is considered the gold standard for evaluation of tissue fibrosis. Biopsy specimens of myocardial, renal, and pulmonary parenchyma were not obtained because of the invasiveness of those procedures and the fact that PIIINP was studied in a population that included hospital patients. It is a well-known fact that fibrosis is not an inevitable feature of heart disease because the patterns of myocardial remodeling are variable according to the underlying disease process. ${ }^{33}$ Although myocardial fibrosis has been associated with volume overload by some authors, ${ }^{34}$ there is convincing evidence that myocardial fibrosis is rather a minor feature in myocardial remodeling secondary to volume overload. ${ }^{35}$ This could in fact explain why serum PIIINP concentrations were within reference range in dogs with cardiac insufficiency secondary to DVD (leading to volume overload), but not in the group of dogs with CMP.

However, in BALF, mean PIIINP concentration was significantly higher in dogs with CBP, compared with healthy adult control dogs. In the present study, most of the dogs with CBP had eosinophilic BP, a disease characterized by eosinophilic infiltration of the pulmonary tissue and bronchial mucosa that has been well described in dogs. ${ }^{10,36}$ High activities of serine proteases and metalloproteinases have been reported in BALF of dogs with eosinophilic BP and seem to be responsible for the extensive destruction and remodeling of the pulmonary tissue. ${ }^{37,38}$ The results of our study indicated that an increased collagen type III turnover resulting from the extensive pulmonary remodeling in this condition may be well reflected by increased PIIINP concentrations in BALF. Nevertheless, there was considerable overlap between PIIINP concentrations in BALF of healthy and diseased adult dogs. A few dogs had extremely high BALF PIIINP concentrations. Whether BALF PIIINP concentration can be correlated to severity of BP still needs to be determined. Growing dogs had significantly higher BALF PIIINP concentrations, compared with adult dogs, reflecting increased collagen type III turnover related to growth.

Our data suggest that the radioimmunoassay based on human PIIINP is accurate and sufficiently precise for measurement of canine PIIINP in serum and BALF. In healthy adult dogs, serum PIIINP concentration appears to be strongly positively correlated with weight and mildly negatively correlated with age. Serum PIIINP concentration in dogs was not notably affected by renal insufficiency, heart disease, or chronic pulmonary disease, but high BALF PIIINP concentrations were detected in dogs with CBP. We therefore believe that BALF PIIINP concentration could be a promising marker of lung disease and merits further investigation in a larger population of dogs with various bronchopulmonary pathologies. However, the naturally higher PIIINP concentrations in serum and BALF in healthy growing dogs, compared with adult dogs, might limit the usefulness of PIIINP as a marker of fibrosis in these young animals.

\footnotetext{
a. BD Vacutainer Systems, Plymouth, UK.

b. Centrifuge Hettich EBA Universal II, Tuttlingen, Germany.
}

c. Eppendorf tubes, Eppendorf-Nethler-Hinz GmbH, Hamburg, Germany.

d. UniQ PIIINP radioimmunoassay, Orion Diagnostics, Espoo, Finland.

e. Phosphate-buffered saline solution $\left(0.3 \mathrm{~g}\right.$ of $\mathrm{NaH}_{2} \mathrm{PO}_{4}, 1.1 \mathrm{~g}$ of $\mathrm{Na}_{2} \mathrm{HPO}_{4}$, and $9.0 \mathrm{~g}$ of $\mathrm{NaCl} / \mathrm{L}$ ad 1000 Aqua bidestilata), adjusted with $1 \mathrm{~N} \mathrm{HCl}$ or $1 \mathrm{~N} \mathrm{NaOH}$ to $\mathrm{pH} 7.4$, Merck, Darmstadt, Germany. f. Saline $(0.9 \% \mathrm{NaCl})$ solution, Merck KgaA, Darmstadt, Germany.

\section{References}

1. Bitterman PB, Henke CA. Fibroproliferative disorders Chest 1991;99(suppl 3):81S-84S

2. Risteli L, Risteli J. Analysis of extracellular matrix proteins in biological fluids. Methods Enzymol 1987;145:391-411.

3. Fessler JH, Fessler LI. Biosynthesis of procollagen. Annu Rev Biochem 1978;47:129-162.

4. Pierard D, Nusgens BV, Lapiere CM. Radioimmunoassay for the amino-terminal sequences of type III procollagen in human body fluids measuring fragmented precursor sequences. Anal Biochem 1984;141:127-136.

5. Low RB, Cutroneo KR, Davins GS, et al. Lavage type III procollagen N-terminal peptides in human pulmonary fibrosis and sarcoidosis. Lab Invest 1983;48:755-759.

6. Jensen LT. The aminoterminal propeptide of type III procollagen. Studies on physiology and pathophysiology. Dan Med Bull 1997:44:70-78.

7. Risteli L, Risteli J. Non-invasive methods for detection of organ fibrosis. In: Roijkind M, ed. Connective tissue in health and disease. Boca Raton, Fla: CRP Press, 1990;61-98.

8. Trivedi P, Cheeseman P, Portmann B, et al. Variation in serum type III procollagen peptide with age in healthy subjects and its comparative value in the assessment of disease activity in children and adults with chronic active hepatitis. Eur J Clin Invest 1985;15:69-74.

9. Risteli J, Niemi S, Trivedi P, et al. Rapid equilibrium radioimmunoassay for the amino-terminal propreptide of human type III procollagen. Clin Chem 1988;34:715-718

10. Leroy V, Monier F, Bottari S, et al. Circulating matrix metalloproteinases 1, 2, 9 and their inhibitors TIMP-1 and TIMP-2 as serum markers of liver fibrosis in patients with chronic hepatitis C: comparison with PIIINP and hyaluronic acid. Am J Gastroenterol 2004;99:271-279.

11. Niemelä O, Risteli J, Blake JE, et al. Markers of fibrogenesis and basement membrane formation in alcoholic liver disease Relation to severity, presence of hepatitis, and alcohol intake. Gastroenterology 1990;98:1612-1619.

12. Hochweiss S, Fruchtman S, Hahn EG, et al. Increased serum procollagen III aminoterminal peptide in myelofibrosis. Am J Hematol 1983;15:343-351.

13. Lee YJ, Shin KC, Kang SW, et al. Type III procollagen N-terminal propeptide, soluble interleukin-2 receptor, and von Willebrand factor in systemic sclerosis. Clin Exp Rheumatol 2001;19:69-74.

14. Zhu GG, Stenback F, Risteli L, et al. Organization of type III collagen in benign and malignant ovarian tumors. An immunohistochemical study. Cancer 1993;72:1679-1684.

15. Uusimaa P, Risteli J, Niemela M, et al. Collagen scar formation after acute myocardial infarction: relationships to infarct size, left ventricular function, and coronary artery patency. Circulation 1997;96:2565-2572

16. Klappacher G, Franzen P, Haab D, et al. Measuring extracellular matrix turnover in the serum of patients with idiopathic or ischemic dilated cardiomyopathy and impact on diagnosis and prognosis. Am J Cardiol 1995;75:913-918.

17. Zannad F, Dousset B, Alla F. Treatment of congestive heart failure: interfering the aldosterone-cardiac extracellular matrix relationship. Hypertension 2001;38:1227-1232.

18. Keller F, Rehbein C, Schwarz A, et al. Increased procollagen III production in patients with kidney disease. Nephron 1988; 50:332-337.

19. Honkanen E, Froseth B, Gronhagen-Riska C. Serum hyaluronic acid and procollagen III amino terminal propeptide in chronic renal failure. Am J Nephrol 1991;11:201-206.

20. Teppo AM, Tornroth T, Honkanen E, et al. Urinary aminoterminal propeptide of type III procollagen (PIIINP) as a marker of 
interstitial fibrosis in renal transplant recipients. Transplantation 2003; 75:2113-2119

21. Entzian P, Huckstadt A, Kreipe H, et al. Determination of serum concentrations of type III procollagen peptide in mechanically-ventilated patients. Am Rev Respir Dis 1990;142:1079-1082.

22. Lammi L, Kinnula V, Lähde S, et al. Propeptide levels of type III and type I procollagen in the serum and bronchoalveolar lavage fluid of patients with pulmonary sarcoidosis. Eur Respir J 1997;10:2725-2730.

23. Clercx C, Peeters D, Snaps F, et al. Eosinophilic bronchopneumopathy in dogs. J Vet Intern Med 2000;14:282-291.

24. Jones S. Structure, biosynthesis and disorders of collagen: a review. Bull Am Soc Vet Clin Pathol 1976;5:4-16.

25. Jensen LT, Hendriksen JH, Risteli J, et al. Fate of circulating amino-terminal propeptide of type III procollagen in conscious pigs. Am J Physiol 1993;265:R139-R145.

26. Madsen JS, Svalastoga E, Jensen L. Serum concentration of procollagen type III aminoterminal peptide in growing dogs with hip dysplasia. Acta Vet Scand 1995;36:157-160.

27. Eto N, Yoshino J, Inui K, et al. Fluctuations of the fibrosis markers with aging. Nippon Ronen Igakkai Zasshi 2002;39:176-180.

28. Laron Z, Klinger B, Jensen LT, et al. Biochemical and hormonal changes induced by one week of administration of rIGF-I to patients with Laron type dwarfism. Clin Endocrinol (Oxf) 1991;35:145-150.

29. Sartorio A, Agosti F, Marazzi N, et al. Combined evaluation of resting IGF-I, N-terminal propeptide of type III procollagen (PIIINP) and C-terminal cross-linked telopeptide of type I collagen (ICTP) lev- els might be useful for detecting inappropriate GH administration in athletes: a preliminary report. Clin Endocrinol (Oxf) 2004;61:487-493.

30. Eigenmann JE, Amador A, Patterson DF. Insulin-like growth factor I levels in proportionate dogs, chondrodystrophic dogs and in giant dogs. Acta Endocrinol (Copenh) 1988;118:105-108.

31. Rasmussen MH, Jensen LY, Andersen T, et al. Collagen metabolism in obesity: the effect of weight loss. Int J Obes Relat Metab Disord 1995;19:659-663.

32. Risteli J, Risteli L. Analysing connective tissue metabolites in human serum. Biochemical, physiological and methodological aspects. J Hepatol 1995;22(suppl 2):77-81.

33. Swynghedauw B. Molecular mechanisms of myocardial remodeling. Physiol Rev 1999;79:215-262.

34. Brower GL, Janicki JS. Contribution of ventricular remodeling to pathogenesis of heart failure in rats. Am J Physiol Heart Circ Physiol 2001;280:H674-H683.

35. Weber KT. Monitoring tissue repair and fibrosis from a distance. Circulation 1997;96:2488-2492.

36. Clercx C, Peeters D, German AJ, et al. An immunologic investigation of canine eosinophilic bronchopneumopathy. J Vet Intern Med 2002;16:229-237.

37. Rajamäki MM, Jarvinen AK, Sorsa T, et al. Collagenolytic activity in bronchoalveolar lavage fluid in canine pulmonary eosinophilia. J Vet Intern Med 2002;16:658-664.

38. Rajamäki MM, Jarvinen AK, Sorsa T, et al. Clinical findings, bronchoalveolar lavage fluid cytology and matrix metalloproteinase2 and -9 in canine pulmonary eosinophilia. Vet J 2002;163:168-181. 